\title{
Los grupos de interés en la programación de producción de un sistema de manufactura "Job Shop"
}

\author{
$\checkmark$ Germán Augusto Coca Ortegón ${ }^{1}$ \\ OMAR DANILO CASTRILLÓN GÓMEZ \\ SANTIAGo RUIZ HERRERA ${ }^{2}$
}

\section{Resumen}

En este artículo, se integran durante el proceso de programación de un sistema de manufactura tipo "Job Shop", algunas expectativas de los siguientes grupos de interés: proveedores, sociedad, colaboradores de la organización, clientes y accionistas. De esta forma, se diseña un proceso de evaluación multiobjetivo, por medio del cual se pretende la minimización simultánea del conjunto de variables presentadas a continuación: tiempo proceso, emisiones dióxido de carbono, nivel de fatiga, factor global desperdicio y costos de producción. Asimismo, se expresa que el método propuesto, se encuentra fundamentado en la selección de aquellos individuos, cuyo grado de cercanía a determinados puntos de referencia, sea el máximo posible. Es así como, el desempeño del método anterior (método puntos referencia), se coteja con el desempeño de cierto método multiobjetivo, basado en análisis de subgrupos (método subgrupos). Al respecto, se observa que el primer método muestra, mayor capacidad para detectar la fluctuación estadística inherente a los datos. De este modo, se establece que el "método puntos referencia" supera el desempeño del "método subgrupos", en cuanto al comportamiento de los parámetros “coeficiente de variación” y “rango", para el caso de 4 (tiempo proceso, emisiones dióxido carbono, nivel fatiga, costos producción) de las 5 variables analizadas.

Palabras clave: métodos multiobjetivo, costos de producción, fatiga, grupos de interés, Job Shop.

1 Universidad EIA. Envigado, Colombia.

2 Universidad Nacional de Colombia. Manizales, Colombia.

Autor de correspondencia: Coca Ortegón, G.A. (Germán Augusto): Universidad EIA. Alto de las Palmas $\mathrm{Km} 2$ + 200. Vía aeropuerto José María Córdova. Teléfono: 3104590705.

Correo electrónico: german.coca@eia.edu.co
Historia del artículo:

Artículo recibido: 8-IV-2018 / Aprobado: 30-V-2019

Disponible online: 3 de julio de 2019

Discusión abierta hasta marzo de 2021 


\title{
Stakeholders in the production scheduling of a "Job Shop" manufacturing system
}

\begin{abstract}
In this paper, during the programming process of a "Job Shop" manufacturing system, some expectations of the following stakeholders, are integrated: suppliers, society, employees of the organization, clients and shareholders. In this way, a multiobjective evaluation process is designed. This process lets to minimize, the set of variables presented below: makespan time, carbon dioxide emissions, fatigue level, waste global factor and production costs. Likewise, it is expressed that the proposed method is based on the selection of those chromosomes, whose degree of proximity to certain reference points, is the maximum possible. In this respect, the performance of the previous method (method: reference points), is compared with the performance of a certain multiobjective method, based on subgroup analysis (method: subgroups). Thus, it is observed that the first method shows, greater capacity to detect the statistical fluctuation inherent to the data. In this way, it is established that the "method: reference points" exceeds the performance of the "method: subgroups", in terms of the behavior of the parameters "coefficient of variation" and "rank", in the case of 4 (makespan time, carbon dioxide emissions, fatigue level, production costs) of the 5 variables analyzed.
\end{abstract}

Keywords: multiobjective methods, production costs, fatigue level, stakeholders, Job Shop.

\section{Stakeholders na programação de produção de um sistema de manufatura "Job Shop"}

\section{Resumo}

Neste trabalho, durante o processo de programação de um sistema de manufatura "Job Shop", são integradas algumas expectativas das seguintes partes interessadas: fornecedores, sociedade, funcionários da organização, clientes e acionistas. Desta forma, um processo de avaliação multiobjetivo é projetado. Este processo permite minimizar o conjunto de variáveis apresentadas abaixo: tempo de expansão, emissões de dióxido de carbono, nível de fadiga, fator global de resíduos e custos de produção. Da mesma forma, é expresso que o método proposto é baseado na seleção desses cromossomos, cujo grau de proximidade a certos pontos de referência é o máximo possível. A este respeito, o desempenho do método anterior (método: pontos de referência) é comparado com o desempenho de um determinado método multiobjetivo, baseado na análise de subgrupos (método: subgrupos). Assim, observa-se que o primeiro método mostra, maior capacidade de detectar a flutuação estatística inerente aos dados. Desta forma, estabelece-se que o "método: pontos de referência" excede o desempenho do "método: subgrupos", em termos do comportamento dos parâmetros "coeficiente de variação" e "hierarquia", no caso de 4 (tempo de preparação, emissões de dióxido de carbono, nível de fadiga, custos de produção) das 5 variáveis analisadas.

Palavras-chave: métodos multiobjetivo, custos de produção, nível de fadiga, stakeholders, Job Shop. 


\section{Introducción}

Las organizaciones logran crecer de manera sostenible, a partir de la apropiada gestión de sus relaciones con diversos grupos de interés (Niemi y Pekkola, 2017). Asimismo, se expresa que los procesos de investigación de los sistemas de producción "Job Shop", se han orientado tradicionalmente, hacia el análisis de un conjunto de aspectos asociados con la eficiencia de las operaciones (Shen et al., 2014). En consecuencia, se afirma que la programación del tipo de sistema de manufactura examinado, presenta especial énfasis en la evaluación de indicadores que permiten satisfacer las expectativas de los accionistas y clientes, desconociendo la importancia de cumplir las expectativas de otros grupos de interés: sociedad, colaboradores de la organización, proveedores, entre otros. De este modo, se propone en el presente artículo minimizar, el comportamiento de las siguientes variables, durante la programación de producción de un sistema tipo "Job Shop" de una compañía perteneciente al sector metalmecánico: emisiones equivalentes de dióxido de carbono $\left(\mathrm{CO}_{2}\right)$ en el sistema productivo y en sus sistemas soporte, nivel de fatiga, factor global de desperdicio, costos de producción y tiempo de proceso.

Con relación a las variables mencionadas en el párrafo previo, se señala que su determinación procede de la interpretación de los resultados obtenidos después de aplicar ciertos métodos generales de diagnóstico, cuya utilización es habitual en los procesos de mejora de la empresa observada. Respecto a los métodos en cuestión, se expone que son estructurados y aprobados por competentes organismos internacionales y a la vez, son implementados por organizaciones localizadas en diferentes sitios del mundo. Es así como, se registran a continuación, las denominaciones de dichos métodos de diagnóstico: evaluación de aspectos ambientales ((He et al., 2018), (ISO 14001:2015, 2015)), evaluación de matriz de riesgos industriales ((Abbas et al., 2018), (ISO 45001:2018)) y evaluación de prioridades competitivas de fabricación (Boyer y Lewis,
2002). Con base en lo descrito, se establece que cada compañía y específicamente, cada empresa del sector metalmecánico, podría revisar los resultados provenientes de la aplicación de los métodos de diagnóstico en referencia, determinando de manera consecuente, cuales son las variables prioritarias que deberán modelarse en el campo general del análisis multiobjetivo, con el propósito de representar de forma satisfactoria, los requerimientos o necesidades de sus grupos de interés.

De acuerdo con lo expuesto, se plantea que en el contexto de la compañía objeto de análisis, la evaluación simultánea de las variables identificadas es relevante, debido a los hechos ilustrados enseguida: la dirección de la organización, se ha comprometido a reducir las emisiones equivalentes de dióxido de carbono (evaluación de aspectos ambientales); la causa básica de la accidentalidad laboral, es asignable a la fatiga significativa en los distintos puestos de trabajo (evaluación de matriz de riesgos industriales); la obtención de no conformes de calidad y la ocurrencia de fallos en los equipos, ocasionan el no cumplimiento en las fechas de entrega (evaluación de la prioridad competitiva, tiempo de entrega); controlar los costos de producción, constituye una medida fundamental para alcanzar las metas de rentabilidad de la compañía (evaluación de la prioridad competitiva, costos) y por último, se argumenta que la reducción en los tiempos de proceso podría incrementar la frecuencia de compra de materia prima (beneficio para los proveedores) (evaluación de la prioridad competitiva, flexibilidad) y mejoraría potencialmente, los estándares de servicio desde el área productiva (evaluación de la prioridad competitiva, tiempo de entrega).

A su vez, se denota que la determinación del tiempo de procesamiento sub-óptimo de los sistemas "Job Shop Flexible", corresponde a la categoría de problemas del tipo "NP duro", puesto que dada la existencia de " $F$ " órdenes de fabricación y de "T" centros de trabajo, surge la necesidad de evaluar un total de F! ${ }^{\mathrm{T}}$ soluciones posibles (Karimi et al., 2017). Es así como, después de identificar el comportamiento 
de la variable tiempo de proceso, se podrá evaluar el problema multiobjetivo de interés. En este sentido, la revisión de la literatura de corriente principal, permite discriminar las siguientes categorías, en el campo de la programación multiobjetivo de los sistemas "Job Shop":

Evaluación de eficiencia en cuánto a asignación de carga de trabajo. Para el caso, se relacionan entre otros, 6 desarrollos, los cuales buscan minimizar el comportamiento de las variables: tiempo de proceso, total de carga de trabajo y total de carga de trabajo en la operación crítica ((Azadeh et al., 2016), (Huang y Süer, 2015), (Kaplanoglu, 2016), (Ojstersek et al., 2018), (Pérez y Hernández, 2018), (Zhu et al., 2016)).

Evaluación de eficiencia en entrega. Desde esta perspectiva, se observan entre otros, 5 artículos, cuyo enfoque se orienta hacia la minimización del comportamiento de las variables tiempo de proceso y tardanza, con el objetivo de garantizar la entrega oportuna, en el área de producción ((Hao et al., 2017), (Rudy y Zelazny, 2014), (Shivasankaran, 2015), (Singh et al., 2016), (Yan y Yue, 2015)).

Evaluación complementaria de eficiencia productiva. Para el caso, se relacionan entre otros, 5 desarrollos académicos, los cuales analizan la minimización simultánea de las variables tiempo de proceso y costo de manufactura. De esta forma, se pretende asegurar el adecuado control de los costos asignables al proceso de transformación ((Coca et al., 2013), (Geng et al, 2018), (Haider y Mirza, 2015), (Ma et al., 2014), (Robaninejat et al., 2016)).

Evaluación de consumo de energía. Para la categoría se detectan específicamente 5 desarrollos académicos. En relación a lo enunciado, en el primer desarrollo se implementa el algoritmo NSGAII, con el fin de minimizar el comportamiento de las variables tardanza total y consumo de energía eléctrica, en cierto contexto productivo (Liu et al., 2014). En cuanto al segundo artículo, se examina para el mismo contexto revisado en el artículo previo, aquel consumo de energía generado, durante la suspensión periódica del fluido eléctrico por parte del estado (Liu et al., 2015). En el tercer artículo, se evalúa también la situación descrita en el segundo desarrollo, pero se utiliza esta vez con fines de comparación, un algoritmo basado en NSGA-II (Liu et al., 2016). En los artículos, cuarto y quinto, se aprecia la minimización del tiempo de fabricación y del consumo de energía eléctrica, procediendo a definir en lo que respecta particularmente a la segunda variable, aquel consumo de energía relacionado con los diferentes estados de funcionamiento de las máquinas (cambio de herramental, encendido, apagado, en espera, en procesamiento de material) ((Moon y Park, 2014), (Wu y Sun, 2018)). Finalmente, se plantea que en los trabajos revisados, sólo se ilustra la determinación del consumo de energía eléctrica en el sistema de producción, pero no se observa el análisis integral del consumo energético (consumo de energía eléctrica y consumo de combustibles de origen fósil), y su desprendimiento equivalente de $\mathrm{CO}_{2}$, considerando a nivel global, tanto el sistema de producción como sus sistemas o equipos de apoyo (mantenimiento, calderas, sistemas de refrigeración, equipos de control de calidad, entre otros).

Evaluación de la salud ocupacional. Para el caso, se detectan específicamente solo dos desarrollos académicos, bajo los cuales se propone la minimización de las variables: tiempo de proceso y accidentalidad laboral ((Gong et al., 2018), (Ruiz et al., 2012)). De la misma manera, en este artículo se representa la última variable, por medio de una curva de probabilidad horaria, en el respectivo modelo matemático de solución. Sin embargo, en tal desarrollo, no se explora con profundidad el análisis de las causas de la accidentalidad laboral, como tampoco se establece su posible inclusión, en el modelo metaheurístico propuesto.

Complementariamente, se indica que al realizar la consulta en las bases de datos: Web of Science y Scopus, utilizando para este efecto, las ecuaciones de búsqueda: Multibojective and "Job Shop" y Stakeholders and "Job Shop", se detectaron un total de 123 artículos diferentes. Con posterioridad, para 
cada artículo dentro del conjunto de los 123 artículos, se procedió a identificar cada variable analizada (tiempo de proceso, total de carga de trabajo, entre otras). Después, se obtuvo la cantidad porcentual de artículos que incluyen en su tratamiento metodológico cada clase de variable. De este modo, se aprecian enseguida, las participaciones porcentuales de los artículos, en referencia al abordaje de algunas variables identificadas: 95,12\% de los 123 artículos, evalúan la variable tiempo de proceso; $22,76 \%$, el costo de manufactura; $12,19 \%$, el tiempo de flujo; 8,94\%, el tiempo de preparación; $3,25 \%$, el consumo de energía eléctrica y $0,81 \%$, la accidentalidad laboral.

\section{Metodología}

La metodología se estructura como sigue: en los pasos $1 \mathrm{a} 12$, se presenta el método propuesto (método puntos referencia); en el paso 13, se observa la descripción general del método de comparación (método subgrupos); en el paso 14, se indica el esquema de comparación de los métodos en cuestión y finalmente, en el paso 15 , se aprecia el esquema de cotejo del "método puntos referencia" con la forma de operación de la compañía objeto de estudio.
Paso 1. Identificar la configuración del Individuo. La configuración del individuo permite apoyar la ejecución pertinente de dos métodos evolutivos (método puntos referencia y método subgrupos). Ambos métodos detectan parentales (individuos padre) y, obtienen filiales (individuos hijo). Los anteriores individuos mejoran su comportamiento a través de la implementación de las operaciones de cruzamiento, de mutación y de análisis de dominancia realizados en cada ciclo del programa. De esta forma, se espera determinar los individuos de mejor desempeño para el conjunto de variables evaluadas, después de ordenar la población final de respuesta por frentes de Pareto.

Igualmente, se anota que cada individuo está conformado por "g" genes (ver Tabla 1). Al respecto: los genes número "1" a número " $\mathrm{g}-2$ ", contienen el número de las órdenes a ser procesadas; mientras que el gen número "g-1", indica las horas de trabajo al día y a su vez, el gen número "g", señala el número de días a laborar por semana. De esta forma, el gen número "g-1" puede tomar 3 valores: 8, 16 o 24 horas por día; mientras que el gen número "g", puede tomar 2 valores: 6 o 7 días por semana. En la Tabla 1, se observa la configuración del individuo y en la Tabla 2, se aprecia el ejemplo de esta configuración.

TABLA 1. CONFIGURACIÓN DEL INDIVIDUO

\begin{tabular}{|c|c|c|c|c|c|c|c|c|c|c|}
\hline Gen 1 & Gen 2 & Gen 3 & Gen 4 & Gen 5 & Gen 6 & Gen 7 & Gen 8 & Gen g-2 & Gen g-1 & Gen g \\
\hline $\begin{array}{c}\text { Número } \\
\text { primera } \\
\text { orden a } \\
\text { obtener }\end{array}$ & $\begin{array}{c}\text { Número } \\
\text { segunda } \\
\text { orden a } \\
\text { obtener }\end{array}$ & $\begin{array}{c}\text { Número } \\
\text { tercera } \\
\text { orden a } \\
\text { obtener }\end{array}$ & $\begin{array}{c}\text { Número } \\
\text { cuarta } \\
\text { orden a } \\
\text { obtener }\end{array}$ & $\begin{array}{c}\text { Número } \\
\text { quinta } \\
\text { orden a } \\
\text { obtener }\end{array}$ & $\begin{array}{c}\text { Número } \\
\text { sexta } \\
\text { orden a } \\
\text { obtener }\end{array}$ & $\begin{array}{c}\text { Número } \\
\text { séptima } \\
\text { orden a } \\
\text { obtener }\end{array}$ & $\begin{array}{c}\text { Número } \\
\text { octava } \\
\text { orden a } \\
\text { obtener }\end{array}$ & $\begin{array}{c}\text { Número } \\
\text { óltima } \\
\text { obten a }\end{array}$ & $\begin{array}{c}\text { Horas por } \\
\text { Día }\end{array}$ & $\begin{array}{c}\text { Días por } \\
\text { semana }\end{array}$ \\
\end{tabular}

TABLA 2. EJEMPLO CONFIGURACIÓN GENERAL DEL INDIVIDUO

\begin{tabular}{|c|c|c|c|c|c|c|c|c|c|c|}
\hline Gen 1 & Gen 2 & Gen 3 & Gen 4 & Gen 5 & Gen 6 & Gen 7 & Gen 8 & Gen 9 & Gen 10 & Gen 11 \\
\hline $\begin{array}{c}\text { Orden } \\
\text { trabajo } \\
\text { número } \\
7\end{array}$ & $\begin{array}{c}\text { Orden } \\
\text { trabajo } \\
\text { número } \\
3\end{array}$ & $\begin{array}{c}\text { Orden } \\
\text { trabajo } \\
\text { número } \\
6\end{array}$ & $\begin{array}{c}\text { Orden } \\
\text { trabajo } \\
\text { número } \\
1\end{array}$ & $\begin{array}{c}\text { Orden } \\
\text { trabajo } \\
\text { número } \\
8\end{array}$ & $\begin{array}{c}\text { Orden } \\
\text { trabajo } \\
\text { número } 5\end{array}$ & $\begin{array}{c}\text { Orden } \\
\text { trabajo } \\
\text { número } 9\end{array}$ & $\begin{array}{c}\text { Orden } \\
\text { trabajo } \\
\text { número } 2\end{array}$ & $\begin{array}{c}\text { Orden } \\
\text { trabajo } \\
\text { número } 4\end{array}$ & 16 horas & 7 \\
\hline
\end{tabular}


En la Tabla 2, se observa que la orden de trabajo 7 , ingresaría en primer término al proceso de fabricación, luego ingresaría la orden de trabajo 3 y, por último, ingresaría la orden de trabajo 4 . A su vez, el sistema productivo operaría 16 horas al día y 7 días a la semana. Con relación a lo descrito, se señala que cada una de aquellas posibles secuencias de ingreso de tales órdenes de trabajo al sistema de fabricación (ver Tablas $\mathbf{1}$ y $\mathbf{2}$ ), constituye una permutación dentro del conjunto total de permutaciones posibles (9! para el caso de 9 órdenes de trabajo). En consecuencia, para cada permutación bajo análisis, se determinaría en primera instancia durante el proceso de optimización, el respectivo tiempo de proceso expresado en horas (fitness del individuo en horas), mientras que, con posterioridad, se establecería su tiempo equivalente expresado en semanas (fitness del individuo en semanas).

Paso 2. Generar población aleatoria. En este caso, se toma del universo de soluciones, un total de "P" individuos. Es así como, para la situación analizada, existen: 9 órdenes de trabajo, 3 posibles horarios de programación al día (8 horas, 16 horas o 24 horas) y 2 formas de organización de las actividades laborales por semana (6 días o 7 días).

Paso 3. Determinar el valor de las funciones objetivo por individuo. Para los "P" individuos, se determina el valor correspondiente a cada una de las funciones objetivos, así:

Definición de tiempo de procesamiento. Con este propósito, se aplica la Ecuación 1, a aquella información registrada en las Tabla $\mathbf{3}$ (Ver experimentación).

$$
\text { FUNCIÓN }_{\text {TIEMPO PROCESO }}=\operatorname{minim}\left\{\sum_{r=1}^{K} \sum_{s=1}^{L} O_{r s}\right\}
$$

En la Ecuación 1, "K" hace referencia al número de órdenes de trabajo; "L" hace referencia al número de equipos y " $\mathrm{O}_{\mathrm{rs}}$ " hace referencia al tiempo de operación de la orden de trabajo " $r$ " en el equipo "s".

Determinación de desprendimiento de $\mathrm{CO}_{2}$ por equipo o por sistema soporte al sistema producti- vo. Después de identificar el tiempo de proceso por equipo, se procede a establecer aquel tiempo utilizado en la transformación del material, como también se procede a discriminar su tiempo de espera. Luego, se suman los tiempos de transformación y los tiempos de espera. Más adelante, se calcula la cantidad de energía requerida, multiplicando la potencia del equipo en procesamiento o espera (ver Tabla 4), por los valores detectados de cada categoría de tiempo (transformación o espera). Por último, se obtiene el desprendimiento de $\mathrm{CO}_{2}$, al multiplicar la cantidad de energía definida, por el factor de conversión aplicable (ver Tabla 5). Con relación a lo anteriormente expresado, se realizan las siguientes aclaraciones:

Las máquinas $\mathrm{A}, \mathrm{C}, \mathrm{D}$ y $\mathrm{F}$ requieren energía eléctrica para su funcionamiento. La máquina B se alimenta con gas. La máquina $\mathrm{E}$ requiere vapor proveniente de la caldera. La caldera: se alimenta con gas, debe encenderse dos horas antes de iniciar aquellas formas de organización del trabajo que impliquen jornadas laborales de 8 horas o de 16 horas al día, no se apaga sí las jornadas laborales son de 24 horas por día y no presenta cambio de potencia, sí la máquina E se encuentra en espera. El sistema de refrigeración: se abastece de energía eléctrica, trabaja a menor potencia en horario nocturno y no presenta cambios en la misma, durante los tiempos de espera. El sistema de manejo de materiales: está integrado por dos montacargas; el primer montacargas funciona con diésel y se utiliza para transportar, debido a su volumen y geometría, las órdenes de trabajo número: 2, 4, 5 y 8; el segundo tipo de montacargas funciona con gas y permite acarrear las demás órdenes de trabajo; ambos montacargas son utilizados para transportar, las órdenes de fabricación desde sus puntos finales de proceso, hasta el almacén de producto terminado y presentan, diversos tipos de usos en la compañía, los cuales no se documentan en el presente artículo; el tiempo promedio de transporte de las órdenes terminadas al lugar de disposición final en almacén, es de 5 minutos.

Determinación del nivel de fatiga. De acuerdo con los resultados arrojados en la evaluación del 
panorama de riesgos de la organización objeto de estudio, la fatiga se genera como consecuencia de los siguientes hechos: manipulación de elementos pesados, difícil postura al realizar la labor, comportamiento de la temperatura en la zona geográfica de localización de la planta y comportamiento de la humedad relativa en la región. Con base en lo expuesto, se procede a establecer el valor correspondiente al nivel de fatiga, aplicando la técnica utilizada con este propósito, en los estudios relacionados con el examen de métodos de trabajo (Niebel y Freivalds, 2012). De esta manera, bajo el protocolo definido por la técnica en consideración, se efectúa el análisis pertinente de las matrices para asignación de puntos de fatiga, durante el proceso de revisión de las actividades ejecutadas en los puestos de trabajo.

De modo general, se señala que el mecanismo de observación de las matrices en cuestión, implica llevar a cabo las siguientes tareas: discriminar en primera instancia, las condiciones de ejecución física de la labor; con posterioridad, se comparan dichas condiciones con las situaciones descritas en tales matrices; enseguida, se procede a seleccionar aquella situación que presente mayor similitud con las condiciones de trabajo examinadas y por último, se asigna como nivel de fatiga, aquel puntaje registrado en las matrices para cada situación elegida.

En las Tablas 6, 7 y 8, se aprecian respectivamente las características de ejecución de cada operación: peso manipulado en promedio por cada orden de fabricación y tipo de esfuerzo realizado, en cada puesto de trabajo; postura en cada puesto de trabajo; temperatura promedio y humedad relativa promedio para la región durante cada hora del día. Es así como, las anteriores características, se comparan con las matrices correspondientes a la asignación de nivel de fatiga. De esta forma, en las Tablas 9, 10 y 11, se presenta un extracto de las matrices utilizadas en el proceso de determinación del nivel de fatiga para los riesgos evaluados (peso, postura, exposición a temperatura y humedad relativa) (Niebel y Freivalds, 2012).
A partir de lo indicado, se procedió a obtener el valor de la fatiga para los parámetros peso y postura, en el estudio de caso de la compañía analizada. En este sentido, dicho valor se fijó como valor constante por hora y por operación, en la estructura del programa informático diseñado en el presente trabajo. Asimismo, se denota que el programa en mención permite establecer también, el nivel de fatiga para las variables temperatura promedio $\mathrm{y}$ humedad relativa promedio, al considerar sus fluctuaciones por hora y sus equivalencias en puntos de fatiga, a lo largo de las 24 horas del día.

Al tomar referencia en los aspectos descritos en los párrafos previos, para los "P" individuos que integran la población, se identifican los tiempos de transformación y los tiempos de espera. Más adelante, para los tiempos de transformación, se efectúa la sumatoria horaria de los niveles de fatiga detectados. Finalmente, se estima el nivel de fatiga promedio, dividiendo el valor total de fatiga entre el tiempo de proceso (en horas) calculado por cada individuo.

Penalización costos de producción: Para la empresa examinada, se decide incluir en el análisis de costos de producción, aquellos costos que presenten las siguientes características: mayor significancia en su estructura de costos y comportamiento fluctuante, a lo largo de la jornada laboral. De acuerdo con lo definido, se procedió a seleccionar en el contexto de la organización de interés, las categorías de costos de fabricación que se observan enseguida: costos de mano de obra y costos de energía.

Con el propósito de cuantificar los costos de producción, en el proceso de evaluación multiobjetivo, se establecieron curvas de asignación de puntaje, las cuales fluctúan de 0 a 100 puntos, según la hora del día. Desde la perspectiva de los costos de mano de obra, la mencionada asignación de puntaje, se determina con base en los recargos porcentuales pagados por la compañía, sobre el valor del salario en tiempo normal (ver Tabla 12); por ejemplo, se asignan 75 puntos por cada hora, en la jornada laboral de 6 a.m a 10 p.m, en día dominical. Asimismo, en cuanto a los costos por consumo de 
energía, la asignación de puntaje se realiza, considerando las adiciones porcentuales canceladas por la empresa, respecto al valor definido como tarifa mínima de un kilovatio hora (Kwh) para ciertas horas del día (ver Tabla 13).

De forma particular, la obtención de la penalidad por costos de producción, se calcula segregando en primera instancia, los tiempos de transformación de los tiempos de espera, durante el tiempo de proceso de cada individuo. Después, se asigna a los tiempos de transformación horarios, aquellos puntajes correspondientes por costos de mano de obra y por costos de energía, según lo indicado en las Tablas 12 y 13. Por último, se procede a realizar la sumatoria de los puntajes de penalidad previamente hallados.

Penalización factor global de desperdicio: El factor global de desperdicio, permite integrar las métricas de los siguientes factores: factor de desperdicio y factor de no utilización. El factor de desperdicio mide la relación entre las pérdidas de material en fabricación (pérdidas de material originadas en la obtención de productos no conformes, desperdicio normal del proceso y pérdidas de material causadas por diversos errores, en las etapas de procesamiento), respecto a la cantidad total de material que ingresa a fabricación. El factor de no utilización, hace referencia a la relación entre los tiempos perdidos en planta debido a preparaciones $\mathrm{y}$ fallos de equipo, respecto al tiempo total programado en manufactura.

Es así como, se señala que la organización objeto de estudio, con base en el análisis de su información histórica, definió el comportamiento horario aplicable a los factores de desperdicio y de no utilización. De esta manera, la probabilidad inherente a tales factores, se transforma en puntos de penalidad por hora (ver Tabla 14).

En consecuencia con lo anterior, la penalización correspondiente al factor global de desperdicio, se obtiene así: en primer término, se discriminan los tiempos de transformación y los tiempos de espera; luego, se procede a identificar durante los tiempos de transformación, aquel comportamien- to horario de la penalidad, tanto para el factor de desperdicio como para el factor de no utilización; después, por cada hora asignable a los tiempos de transformación, se multiplica el valor de penalización de desperdicio por el valor de penalización de no utilización; finalmente, se suman las penalizaciones horarias que se calcularon en el paso previo.

Paso 4. Obtener y almacenar puntos extremos de referencia. Se genera y evalúa en primera instancia, una población de tamaño "P". Enseguida, se identifican en el conjunto integrado por la población anterior y a su vez, integrado también por la población obtenida en el paso 2, aquellos puntos extremos de cada una de las cinco funciones objetivo. Más adelante, los puntos extremos detectados y sus promedios, se almacenan en una matriz de referencia (individuo de valores máximos, individuo de valores promedio e individuo de valores mínimos), la cual será utilizada en el paso 9 de la presente metodología. Por último, se aclara que el paso 4 , solo se ejecuta en el primer ciclo del programa.

Paso 5. Normalizar la población identificada en el Paso 2. Con el propósito de normalizar los "P" individuos que integran la población, se aplican las siguientes expresiones:

$$
\begin{gathered}
N\left(Y_{r}\right)_{s}=\left[\frac{C N\left(f u n_{s}\right)}{\sum_{l=1}^{U} C N\left(f u n_{l}\right)}\right]\left[\frac{\text { fun }_{s}^{\text {máximo }}-f_{s}\left(y_{r}\right)}{\text { fun }_{s}^{\text {máximo }}-f_{s} \text { mínimo }_{s}}\right] \\
N\left(Y_{r}\right)=\sum_{l=1}^{U} N\left(Y_{r}\right)_{s}
\end{gathered}
$$

En la Ecuación 2: fun ${ }_{\mathrm{s}}^{\text {máximo: }}$ Máximo valor de cada función en los "P" individuos; fun ${ }_{s}^{\text {mínimo: }}$ Mínimo valor de cada función en los "P" individuos; fun $_{\mathrm{s}}\left(\mathrm{y}_{\mathrm{r}}\right)$ : Valor de la función fun ${ }_{\mathrm{s}}$ para el individuo $\mathrm{Y}_{\mathrm{r}}$; $\mathrm{CN}\left(\right.$ fun $_{\mathrm{s}}$ ): Coeficiente de normalización de la función fun $_{\mathrm{s}}$ (El valor del coeficiente de normalización para cada función, lo establece el equipo directivo de la compañía. Este valor puede fluctuar desde 0 hasta 100 puntos. Los valores asignados para el caso de estudio, se registran en la Tabla 15); CN(fun $)$ : Coeficiente de normalización de las "U" funciones objetivo (la sumatoria del coeficiente de normalización para 
las funciones objetivo es igual a 1); $\mathrm{U}$ : Total funciones objetivo; $\mathrm{N}\left(\mathrm{Y}_{\mathrm{r}}\right)_{\mathrm{s}}$ : Normalización individuo en cada función; $\mathrm{Y}_{\mathrm{r}}$ : Individuo en la población de tamaño "P". Después de calcular $\mathrm{N}\left(\mathrm{Y}_{\mathrm{r}}\right)_{\mathrm{s}^{\prime}}$, se obtiene el valor representado en la Ecuación 3. En la Ecuación 3: $N\left(Y_{r}\right)=$ Normalización del individuo "r" (este valor fluctúa de 0 a 100 puntos) (Deb y Jain, 2014).

Con posterioridad, los individuos que integran la población "P", se ordenan a nivel descendente de acuerdo con el valor tomado por la variable $\mathrm{N}\left(\mathrm{Y}_{\mathrm{r}}\right)$. Luego, se eliminan el $20 \%$ de los individuos con menor valor en tal variable $\mathrm{N}\left(\mathrm{Y}_{\mathrm{r}}\right)$.

Paso 6. Completar población de tamaño 2P. Sobre los $0,8 \mathrm{P}$ individuos que integran la población, se realizan las siguientes operaciones:

- Operación de cruzamiento. La operación de cruzamiento, se ejecuta con una probabilidad de $92 \%$. Es así como, se identifican 1,1P individuos. En consecuencia, con lo anterior, la población crece hasta un tamaño de 1,9P Individuos.

- Operación de mutación. Esta operación se efectúa con una probabilidad de $4 \%$. Al respecto, se determinan 0,05P Individuos, lo cual permite incrementar el tamaño de la población hasta 1,95P Individuos.

- Generación aleatoria de individuos. Para el caso, se pretende mantener características de diversidad en la población. Con este propósito, se procede a generar aleatoriamente, la cantidad necesaria de individuos hasta completar 2P individuos.

Paso 7. Identificar frentes de Pareto. Para la identificación de los frentes de Pareto, se aplica el concepto de "no dominancia". De este modo, se comparan aquellos valores establecidos para un individuo en sus diferentes funciones objetivo, respecto a los valores encontrados para los demás individuos, en las mismas funciones objetivo. En referencia a lo indicado, se expresa que sí el individuo en consideración, presenta menores valores en las diferentes funciones, se categoriza como "no dominado". Con base en la argumentación anterior, se señala que el primer frente de Pareto está constituido por un conjunto de individuos, cuyos valores en las diferentes funciones objetivo, cumplen la condición de ser menores al compararse con aquellos valores tomados por los demás individuos en la población, pero no cumplen tal condición al cotejarse entre ellos mismos. Igualmente, se menciona que una vez estructurado el primer frente de Pareto, debe segregarse de la población, con el objetivo de avanzar en el proceso correspondiente a la conformación de frentes. Luego, se obtiene y se separa de la población el segundo frente. Es así como, el proceso continúa hasta obtener el último frente.

Paso 8. Conformar nueva población tamaño P. La población tamaño "P", se define por medio del acopio en etapas sucesivas de los diversos frentes. De esta manera, se toman en primera instancia, aquellos individuos que integran el frente número uno. A su vez, se enuncia que el proceso continúa, anexando cada vez un nuevo frente. Con relación a lo descrito, se denota que si al momento de integrar cada nuevo frente, logra obtenerse de forma exacta la población tamaño P, finaliza este paso de la metodología. En consecuencia, el protocolo metodológico se retoma en el paso 11. Por otro lado, sí al momento de integrar determinado frente, se supera el tamaño poblacional $\mathrm{P}$, es necesario seleccionar individuos del frente en cuestión, hasta completar $\mathrm{P}$ individuos. Al respecto, el protocolo metodológico continúa en el paso 9.

Paso 9. Actualizar puntos extremos de referencia para selección de individuos. En primer término, se identifican los puntos extremos (valores máximos y mínimos) de las funciones objetivo, correspondientes a aquellos individuos que integran la población establecida en el paso 6. Enseguida, se comparan dichos puntos, con aquellos valores extremos (valores máximos y mínimos) que se encuentran registrados en la matriz obtenida en el paso 4 (primer ciclo del programa) o en la misma matriz modificada, después de ejecutar el paso actual (ciclos del programa diferentes al primer ciclo). Más adelante, se verifica que los valores registrados en tal matriz, cumplen la condición de continuar siendo puntos 
extremos, dentro del conjunto de datos comparados. Por último, se ilustra que, en caso de no cumplirse la condición previamente planteada, se procede a reemplazar los valores pertinentes de la matriz en cuestión, por aquellos valores extremos de la población caracterizada en el paso 6.

Paso 10. Seleccionar individuos del último frente hasta completar la población de tamaño $\mathrm{P}$. En este caso, se determinan las distancias geométricas desde los diferentes individuos que conforman aquel frente por medio del cual, se puede completar la población tamaño $\mathrm{P}$, hasta cada uno de los puntos registrados en la matriz obtenida en el paso 9. De esta forma, se ordenan a nivel ascendente dichas distancias. Después, se procede a elegir del listado anterior, la cantidad necesaria de individuos que permitan completar la población tamaño $\mathrm{P}$ y que presenten a la vez, la menor distancia hasta cualquiera de los puntos de referencia.

Paso 11. Completar "K" ciclos de la metodología. Al definir la población de interés, se repite la metodología desde el paso 3, durante $\mathrm{K}$ ciclos.

Paso 12. Ordenar por frentes de Pareto la población final de respuesta. La población final de respuesta se sistematiza en frentes de Pareto, de acuerdo con lo indicado en el paso 7.

Paso 13. Descripción de método de comparación (método subgrupos) (Coca et al., 2013). El método de comparación fue estructurado para el análisis de 3 variables: tiempo de proceso, costos de mano obra y fracción defectuosa. Este método, utiliza las siguientes etapas: generar población aleatoria de tamaño "P"; dividir la población en "N" subgrupos (uno por función objetivo); cuantificar cada subgrupo en una función específica; ordenar de forma ascendente cada subgrupo, de acuerdo con la función analizada; dividir cada subgrupo en tres intervalos uniformes; extraer aleatoriamente de cada intervalo: "0,5P / ( 3 segmentos $\mathrm{x}$ " $\mathrm{N}$ " funciones objetivo)" individuos; obtener a partir del cruzamiento de los 0,5P individuos detectados, 0,4P individuos; obtener por mutación de los 0,9P individuos definidos, 0,1P individuos; repetir durante " $\mathrm{K}$ " veces, desde el paso relacionado con la división de la población en "N" subgrupos. Por último, clasificar la población resultante en frentes de Pareto.

Paso 14. Comparar los resultados de aplicar el "método puntos referencia" y el "método subgrupos". Para el caso, se obtienen los resultados de procesar la información, utilizando con este propósito, los protocolos correspondientes a cada método de análisis. De este modo, se procede a comparar los resultados asignables a la aplicación de los métodos de interés, estableciendo cuál de ellos presenta mayor capacidad de generar respuestas diversas, en el campo relacionado con la evaluación multi-objetivo.

Paso 15. Comparar el método propuesto y el método de operación actual de la compañía analizada. En este paso, se comparan los resultados de aplicar el método propuesto, respecto a la forma de operar de la organización evaluada. En referencia a lo mencionado, se explica que tal organización, opera actualmente utilizando la regla de programación “tiempo de operación más larga" (TOL), pero no realiza análisis multi-objetivo.

\section{Experimentación}

La experimentación se presenta en dos subsecciones, en la primera de ellas se describe el origen de la información, mientras que, en la segunda se aprecia su respectivo proceso de registro.

\section{Subsección 1. Origen de la} información

En la Tabla 2A, se observan las diferentes categorías de información requeridas, su utilidad y su método de determinación. Igualmente, se expresa que los datos necesarios se recolectaron entre los meses de abril a octubre de 2016. Además, se menciona que solamente la variable, "factor global de desperdicio", está relacionada con información de tipo histórico. Al respecto, se aclara que tal información, no se registra en este trabajo, debido a que la compañía decidió suministrar de manera específica su valor promedio. 
TABLA 2A. INFORMACIÓN REQUERIDA, UTILIDAD Y MÉTODO DE DETERMINACIÓN

\begin{tabular}{|c|c|c|}
\hline Información requerida & Utilidad & Método de determinación \\
\hline $\begin{array}{l}\text { Rutas y tiempos de } \\
\text { fabricación de los } \\
\text { pedidos. }\end{array}$ & $\begin{array}{l}\text { Estimar tiempos de procesamiento, con el } \\
\text { propósito de cuantificar otras funciones } \\
\text { objetivo. }\end{array}$ & Entrevista con titular de dirección de planta. \\
\hline $\begin{array}{l}\text { Potencia y fuente por } \\
\text { equipo. }\end{array}$ & $\begin{array}{l}\text { Estimar el consumo de energía por cada } \\
\text { equipo del sistema de producción o del } \\
\text { sistema de apoyo a producción. }\end{array}$ & $\begin{array}{l}\text { Entrevista con titular de dirección de planta } \\
\text { y confirmación mediante recorrido por la } \\
\text { planta de producción. }\end{array}$ \\
\hline $\begin{array}{l}\text { Peso por orden de } \\
\text { fabricación. }\end{array}$ & $\begin{array}{l}\text { Estimar el peso promedio manipulado, en } \\
\text { la programación del conjunto de pedidos } \\
\text { a ingresar en producción. }\end{array}$ & Entrevista con titular de dirección de planta. \\
\hline $\begin{array}{l}\text { Tipo de esfuerzo por } \\
\text { operación. }\end{array}$ & $\begin{array}{l}\text { Identificar el tipo de esfuerzo realizado, } \\
\text { en la programación del conjunto de } \\
\text { pedidos a ingresar en producción. }\end{array}$ & $\begin{array}{l}\text { Observación de los puestos de trabajo en la } \\
\text { planta de producción. }\end{array}$ \\
\hline $\begin{array}{l}\text { Postura por cada puesto } \\
\text { de trabajo. }\end{array}$ & $\begin{array}{l}\text { Determinar el nivel de fatiga generado } \\
\text { por la postura en los diversos puestos de } \\
\text { trabajo. }\end{array}$ & $\begin{array}{l}\text { Observación de los puestos de trabajo en la } \\
\text { planta de producción. }\end{array}$ \\
\hline $\begin{array}{l}\text { Asignación de } \\
\text { puntuación por } \\
\text { fatiga generada por } \\
\text { manipulación de peso, } \\
\text { postura, temperatura y } \\
\text { humedad relativa. }\end{array}$ & $\begin{array}{l}\text { Penalizar los diferentes programas de } \\
\text { producción evaluados, de acuerdo con el } \\
\text { nivel de fatiga. }\end{array}$ & $\begin{array}{l}\text { Reunión para asignación de puntación por } \\
\text { fatiga, en la cual participaron el director de } \\
\text { producción, un supervisor de producción y } \\
\text { dos operarios. }\end{array}$ \\
\hline Fracción defectuosa. & $\begin{array}{l}\text { Penalizar en el indicador "factor global } \\
\text { de desperdicio", los diferentes programas } \\
\text { de producción evaluados, a partir del } \\
\text { porcentaje de fracción defectuosa. }\end{array}$ & Entrevista con titular de dirección de planta. \\
\hline Factor de utilización. & $\begin{array}{l}\text { Penalizar en el indicador "factor global de } \\
\text { desperdicio", los diferentes programas de } \\
\text { producción evaluados, a partir del factor } \\
\text { de utilización por equipo. }\end{array}$ & Entrevista con titular de dirección de planta. \\
\hline $\begin{array}{l}\text { Coeficientes de } \\
\text { normalización por } \\
\text { función objetivo. }\end{array}$ & $\begin{array}{l}\text { Identificar el nivel de importancia de } \\
\text { cada función objetivo. }\end{array}$ & $\begin{array}{l}\text { Reunión para asignación por consenso de } \\
\text { los valores asignados a los coeficientes de } \\
\text { normalización de cada función objetivo, en la } \\
\text { cual participaron: el director de producción, } \\
\text { el director financiero, un supervisor de } \\
\text { producción, un representante del área de } \\
\text { gestión ambiental y los representantes de los } \\
\text { trabajadores, en el comité paritario de salud } \\
\text { ocupacional. }\end{array}$ \\
\hline
\end{tabular}

\section{Subsección 2. Registro de la} información requerida

Enseguida se presenta la información requerida, con el propósito de aplicar tanto el método propuesto (puntos referencia), como el método de comparación (subgrupos).
Rutas y tiempos. En la Tabla 3, se aprecian las rutas de manufactura de cada orden de trabajo, como también se observan sus tiempos de fabricación en cada equipo. Al respecto, se aclara que cada máquina es manejada y controlada por una persona. 


\section{TABLA 3. RUTAS Y TIEMPOS POR ORDEN DE TRABAJO}

\begin{tabular}{|c|c|c|c|c|c|c|c|c|c|c|c|c|}
\hline Orden & \multicolumn{10}{|c|}{ Rutas de manufactura y tiempos de fabricación } \\
\hline 1 & $\mathrm{~B}$ & 14 & $\mathrm{E}$ & 11 & $\mathrm{~A}$ & 09 & $\mathrm{D}$ & 10 & $\mathrm{C}$ & 17 & $\mathrm{~F}$ & 12 \\
\hline 2 & $\mathrm{~F}$ & 10 & $\mathrm{~A}$ & 16 & $\mathrm{~B}$ & 13 & $\mathrm{E}$ & 12 & $\mathrm{D}$ & 11 & $\mathrm{C}$ & 15 \\
\hline 3 & $\mathrm{E}$ & 17 & $\mathrm{C}$ & 07 & $\mathrm{~F}$ & 14 & $\mathrm{~A}$ & 15 & $\mathrm{D}$ & 10 & $\mathrm{~B}$ & 16 \\
\hline 4 & $\mathrm{~A}$ & 12 & $\mathrm{D}$ & 14 & $\mathrm{~B}$ & 18 & $\mathrm{E}$ & 16 & $\mathrm{C}$ & 14 & $\mathrm{~F}$ & 13 \\
\hline 5 & $\mathrm{D}$ & 09 & $\mathrm{~B}$ & 13 & $\mathrm{~F}$ & 17 & $\mathrm{C}$ & 14 & $\mathrm{~A}$ & 12 & $\mathrm{E}$ & 18 \\
\hline 6 & $\mathrm{~B}$ & 15 & $\mathrm{E}$ & 12 & $\mathrm{C}$ & 14 & $\mathrm{D}$ & 11 & $\mathrm{~F}$ & 16 & $\mathrm{~A}$ & 13 \\
\hline 7 & $\mathrm{E}$ & 10 & $\mathrm{~B}$ & 12 & $\mathrm{~A}$ & 18 & $\mathrm{~F}$ & 13 & $\mathrm{C}$ & 12 & $\mathrm{D}$ & 16 \\
\hline 8 & $\mathrm{C}$ & 14 & $\mathrm{D}$ & 15 & $\mathrm{E}$ & 17 & $\mathrm{~A}$ & 15 & $\mathrm{~F}$ & 16 & $\mathrm{~B}$ & 14 \\
\hline 9 & $\mathrm{~F}$ & 08 & $\mathrm{C}$ & 12 & $\mathrm{E}$ & 11 & $\mathrm{~B}$ & 18 & $\mathrm{~A}$ & 12 & $\mathrm{D}$ & 10 \\
\hline
\end{tabular}

TABLA 4. POTENCIA Y FUENTE DE ENERGÍA POR EQUIPO

\begin{tabular}{c|c|c|c|c|c|c|c|c|c}
\hline Equipo & $\begin{array}{c}\text { Tipo } \\
\text { fuente }\end{array}$ & $\begin{array}{c}\text { Potencia } \\
\text { proceso }\end{array}$ & $\begin{array}{c}\text { Potencia } \\
\text { espera }\end{array}$ & Equipo & $\begin{array}{c}\text { Tipo } \\
\text { fuente }\end{array}$ & $\begin{array}{c}\text { Potencia } \\
\text { proceso }\end{array}$ & $\begin{array}{c}\text { Potencia } \\
\text { espera }\end{array}$ & $\begin{array}{c}\text { Potencia } \\
6 \mathrm{am}-10 \mathrm{pm}\end{array}$ & $\begin{array}{c}\text { Potencia } \\
10 \mathrm{pm}-6 \mathrm{am}\end{array}$ \\
\hline $\mathrm{A}$ & Eléctrica & 10,35 & 0,48 & $\mathrm{~F}$ & Eléctrica & 18,5 & 0,81 & - & - \\
\hline $\mathrm{B}$ & Gas & 84,3 & 2,58 & Montacargas 1 & Diésel & 38 & - & - & - \\
\hline $\mathrm{C}$ & Eléctrica & 6,25 & 0,28 & Montacargas 2 & Gas & 42 & - & - & - \\
\hline $\mathrm{D}$ & Eléctrica & 7,38 & 0,37 & Caldera & Gas & 52 & - & - & - \\
\hline $\mathrm{E}$ & Vapor & - & - & Refrigeración & Eléctrica & - & - & 50 & 46 \\
\hline
\end{tabular}

TABLA 5. COEFICIENTES DE CONVERSIÓN A CO2 (KG CO2/ KWH)

\begin{tabular}{c|c|c|c|c|c|}
\hline Fuente & Coeficiente & Fuente & Coeficiente & Fuente & Coeficiente \\
\hline Eléctrica & 0,374 & Gas & 0,2018 & Diésel & 0,2495 \\
\hline
\end{tabular}

TABLA 6. PESO POR ORDEN DE FABRICACIÓN Y TIPO DE ESFUERZO POR OPERACIÓN

\begin{tabular}{c|c|c|c|c|c|c|c|c|c|c|c|c|} 
Orden & 1 & 2 & 3 & 4 & 5 & 6 & 7 & 8 & 9 & Operaciones & A - B - D - F & C-E \\
\hline Peso (Kg) & 52 & 46 & 60 & 68 & 65 & 40 & 70 & 40 & 72 & Tipo de esfuerzo & Intenso & Mediano \\
\hline
\end{tabular}

\begin{tabular}{|c|c|c|c|}
\hline Operaciones & $A-D$ & C-B-F & $\mathrm{E}$ \\
\hline Descripción & Constantemente se estira, inclina y levanta. & A veces se estira, inclina y levanta. & Andando con carga \\
\hline
\end{tabular}

Características equipos. En la Tabla 4, se registran la potencia de cada máquina o equipo (kw) y a su vez, se observa también el tipo de fuente energética utilizada.

Coeficientes de conversión. En la Tabla 5, se observan los coeficientes de conversión, de acuerdo con la fuente energética utilizada, a unidades equivalentes de $\mathrm{CO}_{2}\left(\mathrm{Kg} \mathrm{CO}_{2} / \mathrm{Kwh}\right)$.
Peso y tipo de esfuerzo. En la Tabla 6, se presentan tanto el peso manipulado por tipo de orden de fabricación, como también el tipo de esfuerzo realizado en cada puesto de trabajo.

Postura. En la Tabla 7, se aprecia la postura observada en los puestos de trabajo. 
Temperatura y humedad relativa. En la Tabla $\mathbf{8}$, se registra el valor horario de estas variables.

Puntos por fatiga para el peso. En la Tabla 9, se observa un extracto de la matriz utilizada para la asignación de puntos por fatiga, en cuanto a manipulación de peso.

Puntos por fatiga para la postura. En la Tabla 10, se presenta un extracto de la matriz utilizada para la asignación de puntos por fatiga, en cuanto a la postura.
Puntos por fatiga para la temperatura y para la humedad relativa. En la Tabla 11, se aprecia un extracto para la asignación de puntos por fatiga, aplicable a los parámetros en mención.

Asignación de salarios. En la Tabla 12, se registra la puntuación horaria, de acuerdo con el valor de los recargos sobre el valor del salario en tiempo normal.

Asignación de costos de energía. En la Tabla 13, se observa la puntuación horaria, de acuerdo con el valor de los recargos sobre la tarifa básica de energía.

\section{TABLA 8. TEMPERATURA Y HUMEDAD RELATIVA EN LA REGIÓN}

\begin{tabular}{|l|c|c|c|c|c|c|c|c|}
\hline Horario & $00-01$ & $01-02$ & $02-03$ & $03-04$ & $04-05$ & $05-06$ & $06-07$ & $07-08$ \\
\hline Temperatura $\left({ }^{\circ} \mathrm{C}\right)$ - Humedad relativa $(\%)$ & $11-83$ & $11-83$ & $10-84$ & $10-84$ & $10-84$ & $11-83$ & $12-82$ & $13-81$ \\
\hline Horario & $08-09$ & $09-10$ & $10-11$ & $11-12$ & $12-13$ & $13-14$ & $14-15$ & $15-16$ \\
\hline Temperatura $\left({ }^{\circ} \mathrm{C}\right)-$ Humedad relativa $(\%)$ & $14-80$ & $15-78$ & $16-77$ & $17-76$ & $18-75$ & $18-75$ & $19-74$ & $18-75$ \\
\hline Horario & $16-17$ & $17-18$ & $18-19$ & $19-20$ & $20-21$ & $21-22$ & $22-23$ & $23-24$ \\
\hline Temperatura $\left({ }^{\circ} \mathrm{C}\right)-$ Humedad relaltiva $(\%)$ & $18-75$ & $17-76$ & $16-77$ & $15-78$ & $14-80$ & $13-81$ & $12-82$ & $12-82$ \\
\hline
\end{tabular}

\section{TABLA 9. ASIGNACIÓN DE PUNTOS POR FATIGA (VARIABLE PESO)}

Esfuerzo mediano: puntos para la fuerza ejercida en promedio

\begin{tabular}{c|c|c|c|c|c|c|c|c|c|c}
\hline $\mathrm{Kg}$ & 0 & 0,5 & 1 & 1,5 & 2 & 2,5 & 3 & 3,5 & 4 & 4,5 \\
\hline $0-5$ & $0-15$ & $0-16$ & $0-17$ & $0-18$ & $3-19$ & $6-20$ & $8-21$ & $10-22$ & $12-23$ & $14-24$ \\
\hline
\end{tabular}

\section{TABLA 10. ASIGNACIÓN DE PUNTOS POR FATIGA (VARIABLE POSTURA)}

\begin{tabular}{c|c|c|c|} 
Postura & Puntos & Postura & Puntos \\
\hline Sentado cómodamente & 0 & Sentado incómodamente, o a veces sentado y a veces de pie & 2 \\
\hline
\end{tabular}

\section{TABLA 11. ASIGNACIÓN DE PUNTOS POR FATIGA (VARIABLES TEMPERATURA Y HUMEDAD RELATIVA)}

\begin{tabular}{|c|c|c|c|}
\hline \multirow{2}{*}{ Humedad relativa (\%) } & \multicolumn{3}{|c|}{ Temperatura $\left({ }^{\circ} \mathrm{C}\right)$} \\
\cline { 2 - 4 } & Hasta 23 & De 23 a 32 & Más de 32 \\
\hline Hasta 75 & 0 & $6-9$ & $12-16$ \\
\hline
\end{tabular}

\section{TABLA 12. ASIGNACIÓN DE PUNTUACIÓN POR VALOR DEL SALARIO}

Tipo día y horario - Puntos $\mid$ Hábil y 06 a 22 - 0 | Hábil y 22 a 06 - $35 \mid$ Festivo y 06 a 22 - $75 \mid$ Festivo y 22 a 06 - 110

TABLA 13. ASIGNACIÓN DE PUNTUACIÓN POR VALOR DE LA ENERGÍA

Horario - Puntos
06 a $09-40$
09 a $17-100$
17 a $22-60$

22 a $06-0$ 


TABLA 14. ASIGNACIÓN PUNTUACIÓN FRACCIÓN DEFECTUOSA Y NO UTILIZACIÓN DE EQUIPOS
\begin{tabular}{|l|c|c|c|c|c|c|c|c|}
\hline Horario & $00-01$ & $01-02$ & $02-03$ & $03-04$ & $04-05$ & $05-06$ & $06-07$ & $07-08$ \\
\hline Fracción defectuosa - No utilización & $3-3$ & $3-2$ & $4-2$ & $4-1$ & $3-3$ & $5-4$ & $0-4$ & $1-2$ \\
\hline Horario & $08-09$ & $09-10$ & $10-11$ & $11-12$ & $12-13$ & $13-14$ & $14-15$ & $15-16$ \\
\hline Fracción defectuosa - No utilización & $1-3$ & $1-0$ & $0-6$ & $3-5$ & $3-0$ & $4-3$ & $0-3$ & 2 \\
\hline Horario & $16-17$ & $17-18$ & $18-19$ & $19-20$ & $20-21$ & $21-22$ & $22-23$ & $23-24$ \\
\hline Fracción defectuosa - No utilización & $2-3$ & $3-0$ & $4-2$ & $4-4$ & $4-5$ & $5-5$ & $1-4$ & $3-4$ \\
\hline
\end{tabular}

TABLA 15. COEFICIENTES DE NORMALIZACIÓN POR FUNCIÓN OBJETIVO: CN (fun ${ }_{s}$ )

\begin{tabular}{c|c|c|c|c|c} 
Función & Tiempo de proceso & Emisiones $\mathrm{CO}_{2}$ & Nivel fatiga & Factor global desperdicio & Costos producción \\
\hline $\mathrm{CN}$ (fun $)$ (\%) & 10 & 25 & 25 & 20 & 20 \\
\hline
\end{tabular}

TABLA 16. RESULTADOS GENERALES APLICACIÓN MÉTODO PUNTOS REFERENCIA

\begin{tabular}{|c|c|c|c|c|c|c|c|}
\hline \multirow{3}{*}{$\begin{array}{l}\text { Número } \\
\text { individuo }\end{array}$} & \multirow{3}{*}{$\begin{array}{l}\text { Tiempo } \\
\text { proceso } \\
\text { (horas) }\end{array}$} & \multirow{3}{*}{$\begin{array}{c}\text { Número } \\
\text { horas } \\
\text { día } \\
- \\
\text { Número } \\
\text { días } \\
\text { semana }\end{array}$} & \multicolumn{5}{|c|}{ Grupos de interés y sus variables } \\
\hline & & & $\begin{array}{l}\text { Proveedores } \\
\text { Clientes } \\
\text { Accionistas }\end{array}$ & Sociedad & $\begin{array}{c}\text { Colaboradores } \\
\text { organización }\end{array}$ & $\begin{array}{c}\text { Clientes } \\
\text { Accionistas }\end{array}$ & Accionistas \\
\hline & & & $\begin{array}{l}\text { Tiempo } \\
\text { proceso } \\
\text { (semanas) }\end{array}$ & $\begin{array}{l}\text { Desprendimiento } \\
\qquad \mathrm{CO}_{2}(\mathrm{Kg})\end{array}$ & $\begin{array}{l}\text { Nivel de fatiga } \\
\quad \text { (puntos) }\end{array}$ & $\begin{array}{l}\text { Factor global } \\
\text { desperdicio } \\
\text { (puntos) }\end{array}$ & $\begin{array}{l}\text { Costos } \\
\text { producción } \\
\text { (puntos) }\end{array}$ \\
\hline 3 & 196 & $8-6$ & 4,08 & 14109,26 & 448,73 & 2862 & 6772 \\
\hline 92 & 262 & $24-7$ & 1,56 & 14968,03 & 336,86 & 5436 & 17807 \\
\hline Promedio & 209,82 & & 2,45 & 14285,55 & 438,27 & 4447,25 & 11931,69 \\
\hline
\end{tabular}

Asignación de puntuación por fracción defectuosa y por probabilidad de no utilización de equipos. En la Tabla 14, se presenta la puntuación horaria, de acuerdo con los niveles de fracción defectuosa y de probabilidad de no utilización de equipos.

Coeficientes de normalización por función. En la Tabla 15, se observan aquellos coeficientes asignados por el equipo directivo de la compañía, a las diversas funciones evaluadas.

\section{Resultados y Discusión}

A continuación, se presenta: en primer término, la clasificación de los resultados que se obtuvieron al aplicar ambos métodos; más adelante, se expone el cotejo de los resultados definidos, al implementar los métodos de interés; luego, se ilustra la comparación entre los resultados de ejecutar el "método puntos referencia", respecto a la forma de operar de la empresa y, por último, se desarrolla la discusión de los resultados.

Sistematización resultados "método puntos referencia". De acuerdo con los lineamientos descritos en los pasos 1 a 12 de la metodología, se procede a registrar enseguida, los resultados correspondientes a la aplicación del primer método. Al respecto, se aprecian en la Tabla 16: los grupos de interés y las variables relacionadas con sus expectativas, la caracterización de 2 individuos provenientes de una población total de 100 individuos y asimismo, el promedio de la población para las cinco funciones objetivo.

A su vez, la información correspondiente al número de individuos por frente, se observa a 
continuación: 32 en frente 1, 24 en frente 2, 17 en frente 3, 15 en frente 4 y 12 en frente 5. Complementariamente, se expresa que el equipo de dirección de la compañía, sistematizó por intervalos aquella información específica del primer frente. Para este efecto, se utilizó como medio de clasificación, la variable denominada tiempo de proceso. De este modo, en la Tabla 17 para cada intervalo de tiempo, se indica: las circunstancias de implementación según la naturaleza del mercado, su posible impacto negativo sobre algunos grupos de in- terés y aquellas medidas a tomar, con el propósito de evitar los posibles impactos negativos.

Sistematización resultados "método subgrupos". Con base en los lineamientos establecidos en el paso 13 de la metodología, se procede a registrar en la Tabla 18, los resultados asignables a la ejecución del segundo método. Es así como, se decide implementar para el caso, aquel esquema de presentación ya utilizado, durante el proceso correspondiente, a la síntesis del conjunto de resultados que se obtuvieron, después de aplicar el primer método.

TABLA 17. CLASIFICACIÓN INDIVIDUOS DEL PRIMER FRENTE (MÉTODO PUNTOS REFERENCIA)

\begin{tabular}{|c|c|c|c|}
\hline $\begin{array}{l}\text { Intervalo } \\
\text { tiempo } \\
\text { proceso } \\
\text { (semanas) }\end{array}$ & $\begin{array}{l}\text { Circunstancias de segmentos } \\
\text { de mercado }\end{array}$ & Posibles impactos negativos & $\begin{array}{l}\text { Posibles medidas a } \\
\text { tomar }\end{array}$ \\
\hline $0,92-2$ & $\begin{array}{l}\text { Los segmentos de mercado } \\
\text { crecen de forma sostenida. La } \\
\text { compañía opera a capacidad } \\
\text { máxima. }\end{array}$ & $\begin{array}{l}\text { La fatiga alcanza su mayor valor. La utilización de } \\
\text { la caldera, incrementa las emisiones de } \mathrm{CO}_{2} \text {. Los } \\
\text { costos de producción podrían reducir la utilidad. }\end{array}$ & $\begin{array}{l}\text { Realizar estudios } \\
\text { de: fatiga, uso de } \\
\text { caldera, evaluación } \\
\text { de costos. }\end{array}$ \\
\hline $2-3,5$ & $\begin{array}{l}\text { Los segmentos de mercado } \\
\text { presentan estabilidad. Se } \\
\text { utiliza del } 50 \% \text { al } 70 \% \text { de la } \\
\text { capacidad. }\end{array}$ & $\begin{array}{l}\text { Se detectan programas que generan el mejor } \\
\text { equilibrio, entre las expectativas de los grupos } \\
\text { de interés. Sin embargo, es difícil elegir los } \\
\text { individuos apropiados. }\end{array}$ & $\begin{array}{l}\text { Establecer criterios } \\
\text { para guiar la } \\
\text { selección de } \\
\text { individuos. }\end{array}$ \\
\hline $3,5-5,46$ & $\begin{array}{l}\text { Los segmentos de mercado, } \\
\text { presentan baja demanda. Se } \\
\text { utiliza del } 20 \% \text { al } 50 \% \text { de la } \\
\text { capacidad. }\end{array}$ & $\begin{array}{l}\text { Para el caso, se identifican las siguientes } \\
\text { percepciones: los colaboradores temen ser } \\
\text { despedidos; los accionistas argumentan, la } \\
\text { necesidad de incrementar el uso de la capacidad; } \\
\text { los proveedores expresan la necesidad de que la } \\
\text { compañía aumente, la frecuencia de compra. }\end{array}$ & $\begin{array}{l}\text { Es necesario } \\
\text { incentivar el } \\
\text { aumento de la } \\
\text { demanda en el } \\
\text { mercado. }\end{array}$ \\
\hline
\end{tabular}

TABLA 18. RESULTADOS GENERALES APLICACIÓN MÉTODO SUBGRUPOS

\begin{tabular}{|c|c|c|c|c|c|c|c|}
\hline \multirow{3}{*}{$\begin{array}{l}\text { Número } \\
\text { individuo }\end{array}$} & \multirow{3}{*}{$\begin{array}{l}\text { Tiempo } \\
\text { proceso } \\
\text { (horas) }\end{array}$} & \multirow{3}{*}{$\begin{array}{l}\text { Número } \\
\text { horas } \\
\text { día } \\
- \\
\text { Número } \\
\text { días } \\
\text { semana }\end{array}$} & \multicolumn{5}{|c|}{ Grupos de interés y sus variables } \\
\hline & & & $\begin{array}{c}\text { Proveedores } \\
\text { Clientes } \\
\text { Accionistas }\end{array}$ & Sociedad & $\begin{array}{l}\text { Colaboradores } \\
\text { organización }\end{array}$ & $\begin{array}{c}\text { Clientes } \\
\text { Accionistas }\end{array}$ & Accionistas \\
\hline & & & $\begin{array}{c}\text { Tiempo } \\
\text { proceso } \\
\text { (semanas) }\end{array}$ & $\begin{array}{l}\text { Desprendimiento } \\
\qquad \mathrm{CO}_{2}(\mathrm{Kg})\end{array}$ & $\begin{array}{l}\text { Nivel de fatiga } \\
\quad \text { (puntos) }\end{array}$ & $\begin{array}{c}\text { Factor } \\
\text { global } \\
\text { desperdicio } \\
\text { (puntos) }\end{array}$ & $\begin{array}{c}\text { Costos } \\
\text { producción } \\
\text { (puntos) }\end{array}$ \\
\hline 8 & 176 & $8-6$ & 3,67 & 13832,61 & 499,61 & 2874 & 6608 \\
\hline 83 & 239 & $24-7$ & 1,42 & 14674,54 & 369,16 & 5415 & 17578 \\
\hline Promedio & 194,68 & & 2,29 & 14097,17 & 460,63 & 4403,19 & 12284,62 \\
\hline
\end{tabular}




\begin{tabular}{|c|c|c|c|c|c|c|c|c|}
\hline \multirow{2}{*}{$\begin{array}{c}\text { Métodos } \\
\begin{array}{c}\text { Parámetros } \\
\text { Variables }\end{array}\end{array}$} & \multicolumn{4}{|c|}{ Puntos referencia } & \multicolumn{4}{|c|}{ Subgrupos } \\
\hline & $\begin{array}{l}\text { Coeficiente } \\
\text { variación }\end{array}$ & Máximo & Mínimo & Rango & $\begin{array}{l}\text { Coeficiente } \\
\text { variación }\end{array}$ & Máximo & Mínimo & Rango \\
\hline $\begin{array}{l}\text { Tiempo proceso } \\
\text { (semanas) }\end{array}$ & 0,51 & 5,46 & 0,92 & 4,54 & 0,49 & 4,52 & 0,92 & 3,6 \\
\hline $\begin{array}{l}\text { Desprendimiento } \mathrm{CO}_{2} \\
(\mathrm{Kg})\end{array}$ & 0,04 & 15193,45 & 13295 & 1898,45 & 0,03 & 14794,51 & 13320 & 1474,51 \\
\hline $\begin{array}{l}\text { Nivel de fatiga } \\
\quad \text { (puntos) }\end{array}$ & 0,18 & 572,18 & 335,42 & 236,76 & 0,14 & 572,18 & 368,18 & 204 \\
\hline $\begin{array}{c}\text { Factor global } \\
\text { desperdicio (puntos) }\end{array}$ & 0,26 & 5432 & 2845 & 2587 & 0,27 & 5488 & 2845 & 2643 \\
\hline $\begin{array}{l}\text { Costos de producción } \\
\text { (puntos) }\end{array}$ & 0,32 & 17807 & 6390 & 11417 & 0,31 & 17578 & 6415 & 11163 \\
\hline
\end{tabular}

Enseguida, se aprecia la distribución por frentes del conjunto de 100 individuos obtenidos por medio del método dos: 29 en frente 1, 26 en frente dos, 24 en frente 3 y 21 en frente 4 .

Comparación de los métodos: A partir de lo expresado en el paso 14 de la metodología, se efectúa la comparación pertinente de ambos métodos. Con este objetivo, se procede a evaluar los siguientes parámetros: valores máximos y mínimos, rango y coeficiente de variación. De forma consecuente, en la Tabla 19 se listan aquellos parámetros previamente nombrados.

El contenido de la Tabla 19, permite establecer a partir del análisis de los parámetros, "coeficiente de variación" y rango, la fluctuación inherente a los datos. De este modo, se enuncia que el mejor método corresponde a aquel que presente el mayor valor, en los parámetros previamente nombrados, es decir, que evidencie la capacidad de detectar la máxima fluctuación de las variables en la situación examinada. En consecuencia, se expone que el "método puntos referencia", supera el desempeño del "método subgrupos", en relación al comportamiento del parámetro "coeficiente de variación", de acuerdo con lo indicado enseguida: $4,08 \%$ en lo relativo a la variable tiempo de proceso, $33,33 \%$ en lo pertinente a la variable emisiones de $\mathrm{CO}_{2}, 28,57 \%$ en lo concerniente a la variable nivel de fatiga y $3,22 \%$ en lo aplicable a los costos de producción. Asimismo, se ilustra que la evaluación del comportamiento del parámetro rango, reafirma lo argumentado previamente, es decir, el "método puntos referencia" presenta respecto al "método subgrupos", mayor capacidad para detectar la fluctuación de las variables en el contexto valorado (ver datos rango en Tabla 19).

Además, la comparación de los valores mínimos arrojados por ambos métodos, permite identificar la existencia de las siguientes situaciones: igualdad de los valores mínimos, en el caso de dos variables (tiempo de proceso y factor integral de desperdicio) y mejor desempeño del "método puntos referencia”, en el caso de tres variables. De este modo, el mejor desempeño del último método, se observa en su habilidad de detectar ciertos valores mínimos para las variables desprendimiento de $\mathrm{CO}_{2}$, nivel de fatiga y costos de producción, cuyo comportamiento es inferior al comportamiento mínimo estimado por el "método subgrupos".

Comparación del "método puntos referencia" con el método de operación de la organización. De acuerdo con lo registrado en el paso 15 de la metodología, se desarrolla enseguida la comparación, entre la implementación del primer método, respecto a aquel método aplicado por la empresa objeto de estudio, en el proceso asignable a la programación de sus operaciones. En consecuencia, se enuncia 
que la compañía programa su sistema productivo, utilizando aquella regla de prioridad denominada, tiempo de operación más largo (TOL). De esta manera, se aprecia en la Tabla 20, los resultados de implementar la regla en mención, como también se muestra, la estructura de solución de aquel individuo de menor tiempo de proceso que se generó, luego de haber ejecutado el primer método.

A partir de la información registrada en la Tabla 20, se establece que el primer método, al ser comparado con la regla de programación TOL, presenta mejor desempeño en el comportamiento específico de las siguientes variables: tiempo de proceso (inferior en $12,5 \%$ ), emisiones de $\mathrm{CO}_{2}$ (inferior en $3,13 \%$ ), factor global de desperdicio (inferior en $1 \%$ ) y costos de producción (inferior en 2,91\%). A su vez, la regla TOL muestra mejor desempeño en el comportamiento de la variable nivel de fatiga (inferior en $12,48 \%$ ).

De modo complementario, se aprecia a continuación, el diagrama de Gantt asignable al individuo de menor tiempo de proceso que fue detectado, por medio de la ejecución del primer método.

En la literatura de corriente principal ((Azadeh et al., 2016), (Geng et al., 2018), (Kaplanoğlu, 2016), (Robaninejat et al., 2016), (Shivasankaran, 2015), (Yan y Yue, 2015)) y sus antecedentes, se observa que los procesos de investigación en sistemas "Job Shop", se han orientado prioritariamente hacia la incorporación de diversidad de variables, las cuales se encuentran relacionadas con el campo de la eficiencia técnica. De esta forma, se pretende explicar y mejorar el desempeño económico de tal clase de sistema productivo.

TABLA 20. COMPARACIÓN MÉTODO PUNTOS REFERENCIA Y MÉTODO DE OPERACIÓN DE LA COMPAÑÍA

\begin{tabular}{c|c|c|c|c|c|c|c|}
\hline Método o regla & $\begin{array}{c}\text { Tiempo } \\
\text { proceso } \\
\text { (horas) }\end{array}$ & $\begin{array}{c}\text { Jornada (ho- } \\
\text { ras/día - } \\
\text { días/semana) }\end{array}$ & $\begin{array}{c}\text { Tiempo } \\
\text { proceso } \\
\text { (semanas) }\end{array}$ & $\begin{array}{c}\text { Emisiones } \\
\mathrm{CO}_{2} \\
(\mathrm{Kg})\end{array}$ & $\begin{array}{c}\text { Nivel de } \\
\text { fatiga } \\
\text { (puntos) }\end{array}$ & $\begin{array}{c}\text { Factor global } \\
\text { desperdicio } \\
\text { (puntos) }\end{array}$ & $\begin{array}{c}\text { Costos } \\
\text { producción } \\
\text { (puntos) }\end{array}$ \\
\hline Puntos referencia & 154 & $16-6$ & 1,60 & 13491,20 & 570,01 & 4999 & 6390 \\
\hline TOL & 176 & $16-6$ & 1,83 & 13927,08 & 498,90 & 5049 & 6582 \\
\hline
\end{tabular}

Figura 1. Diagrama de Gantt individuo menor makespan (método puntos referencia)

Fig. 1: Diagrama de Gantt individuo menor makespan (método puntos referencia)

Makespan: 154 horas equivalentes a 1,6 semanas (16 horas día, 6 días por semana)

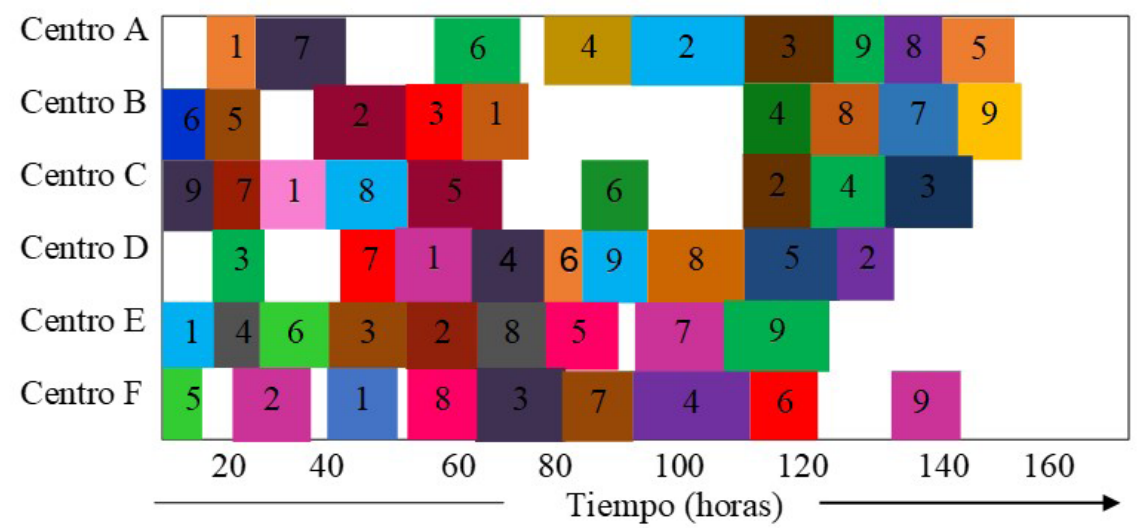


Igualmente, se denota que los procesos de investigación en sistemas "Job Shop", presentan el siguiente grado de profundidad, en cuanto a la evaluación de las expectativas de los grupos de interés: alta en el caso de los accionistas, media en el caso de los clientes y baja, en el caso de la sociedad, los colaboradores de la organización y los proveedores. De manera particular, del conjunto de contribuciones revisadas: 95,12 \% analizan la variable tiempo de proceso (asociada con la reducción de costos y con el cumplimiento en tiempo de entrega), 3,25 \% examinan temas ambientales (consumo de energía eléctrica en el sistema de producción) y $0,81 \%$ evalúan temas sociales (minimización de curva de accidentalidad laboral).

La organización objeto de estudio, debe implementar algún punto localizado en el primer frente de Pareto. De acuerdo con lo anterior, es conveniente que la dirección de la compañía, revise los lineamientos documentados enseguida, con el propósito de guiar el análisis: para aquellos individuos que ocasionan un alto nivel de fatiga, es necesario reducir su efecto, por medio de la contratación de un mayor número de personas; el incremento en costos de producción debe evaluarse, con base en el riesgo financiero de la empresa; debe descartarse en lo posible, la utilización de individuos que impliquen la programación permanente de calderas o de equipos que funcionen a potencias altas y constantes; debe omitirse preferiblemente, la selección de aquellos individuos, cuyos tiempos de proceso (en horas), correspondan a valores máximos, ya que contienen en su estructura, valores representativos de tiempos de espera (durante los tiempos de espera se consume energía y se desprende $\mathrm{CO}_{2}$ equivalente).

En relación al "método puntos referencia", se determinó al cotejarlo con el "método subgrupos", su mejor capacidad para detectar la fluctuación inherente a los datos. De este modo, se expone que el valor del coeficiente de variación establecido, a través de la aplicación del primer método, supera aquel valor obtenido para el mismo parámetro, después de aplicar el segundo método, así: 4,08 \% respecto al tiempo de proceso, 33,33\% respecto a las emisiones de $\mathrm{CO}_{2}, 28,57 \%$ respecto a nivel de fatiga y 3,22 $\%$, respecto a los costos de producción.

Igualmente, se ilustra que los resultados de mejor desempeño, asignables a la aplicación del "método puntos de referencia", se determinaron por medio de la evaluación de los siguientes factores y niveles: tamaño de población de 50 a 300 individuos, número de iteraciones de 40 a 200 ciclos, probabilidad de cruzamiento de $40 \%$ a $100 \%$ y probabilidad de mutación de $1 \%$ a $20 \%$. De esta manera, se procede a presentar enseguida la combinación de parámetros, a través de la cual se obtuvo el comportamiento superior de los indicadores, "coeficiente de variación" y rango: tamaño de población de 100 individuos, 170 iteraciones, probabilidad de cruzamiento de $92 \%$ y probabilidad de mutación de $4 \%$. Por último, se menciona que, en la literatura de corriente principal, no se ha evaluado el conjunto integral de las variables analizadas en el presente artículo; de forma consecuente, no es viable diseñar un estudio comparativo completo.

\section{Conclusiones}

A través del análisis de los resultados y su discusión, se establece: 1) La descripción detallada de la información de entrada y de su sistema de procesamiento, permite que el método propuesto, pueda ser replicado por las personas interesadas en el campo de conocimiento. 2) El proceso de recolección de información, requirió el apoyo mediante entrevista de los titulares de ciertos cargos en la organización analizada; mientras que la definición de factores ponderados para cada una de las funciones objetivo, demandó la realización de reuniones guiadas hasta lograr el consenso necesario. 3) Las expectativas de los colabores de la organización, de la sociedad y de los proveedores, deben incluirse con mayor profundidad, en la conceptualización relacionada con la programación de los sistemas "Job Shop". 4) Las expectativas particulares de los colaboradores de la organización, se han abordado desde el 
análisis básico de la accidentalidad laboral; sin embargo, los conceptos relacionados con el bienestar laboral, no se han analizado con el suficiente rigor. 5) Las expectativas de la sociedad, se han considerado desde el consumo de energía eléctrica en el sistema de producción, pero otros aspectos relacionados con la gestión ambiental, no se han examinado con precisión. 6) En cuanto a los proveedores, se expresa que no se han integrado, sus requerimientos de desarrollo en los programas de producción. 7) A partir del primer frente de Pareto, deben seleccionarse aquellos individuos que generen el mejor equilibrio, entre las expectativas de los diferentes grupos, procediendo a tomar acciones preventivas, cuando alguno de tales grupos, sea negativamente afectado. 8) Tomar puntos de referencia extremos, en el proceso de mejoramiento de la población, permite lograr mayor diversidad en el comportamiento de los individuos detectados.

\section{Agradecimientos}

Se agradece a la Universidad Nacional de Colombia, por su apoyo al proyecto que actualmente se ejecuta, bajo la normatividad del programa de Doctorado en Ingeniería - Industria y Organizaciones y, cuyos resultados parciales se presentan en este artículo.

\section{Referencias}

Abbas, M.; Mneymneh, B. y Khoury, H. (2018). “Assessing on-site construction personnel hazard perception in a Middle Eastern developing country: An interactive graphical approach". Safety Science, vol. 103, pp. 183-196.

Azadeh, A.; Goldansaz, S. y Zahedi, A. (2016). "Solving and optimizing a bi-objective open shop scheduling problem by a modified genetic algorithm". International Journal of Advanced Manufacturing Technology, vol. 85, pp. 1603-1613.

Boyer, K. y Lewis, M. (2002). "Investigating the need for trade-offs in operations strategy". Production and Operations Management, vol. 11, No. 1, pp. 9-20.
Coca, G.; Castrillón, O. y Ruiz, S. (2013). "Metodología basada en los algoritmos VEGA y MOGA para solucionar un problema multi-objetivo en un sistema de producción Job Shop". Revista EIA, vol. 13, pp. 175191.

Deb, K. y Jain, H. (2014). "An Evolutionary Many-Objective Optimization Algorithm Using Reference-PointBased Nondominated Sorting Approach, Part I: Solving Problems With Box Constraints". IEEE Transactions on Evolutionary Computation, vol. 18, No. 4, pp. 577-601.

Geng, Z.; Yuan, J. y Yuan, J. (2018). "Scheduling with or without precedence relations on a serial-batch machine to minimize makespan and maximum cost". Applied Mathematics and Computation, vol. 332, pp. 1-18.

Gong, G.; Deng, Q.; Xuran, G.; Gong, X.; Liu, W. y Ren, Q. (2018). "A new double flexible job-shop scheduling problem integrating processing time, green production, and human factor indicators". Journal of Cleaner Production, vol. 174, pp. 560-576.

Haider, A. y Mirza, J. (2015). "An implementation of lean scheduling in a job shop environment". Advances in Production Engineering \& Management, vol. 10, No. 1, pp. 5-17.

Hao, X.; Gen, M.; Lin, L. y Suer, G. (2017). "Effective multiobjective EDA for bi-criteria stochastic Job-Shop scheduling problem". Journal of intelligent Manufacturing, vol. 28, No. 3, pp. 833-845.

He, B.; Xiao, J. y Deng, Z. (2018). "Product design evaluation for product environmental footprint". Journal of Cleaner Production. vol. 172, pp. 3066-3080.

Huang, J. y Süer, G. (2015). “A dispatching rule-based genetic algorithm for multi-objective job shop scheduling using fuzzy satisfaction levels". Computers \& Industrial Engineering, vol. 86, pp. 29-42.

International Organization for Standardization. (2015). "ISO 14001:2015 Environmental management systems - Requirements with guidance for use".

International Organization for Standardization. (2018). "ISO 45001:2018 Occupational health and safety management systems - Requirements with guidance for use".

Kaplanoğlu, V. (2016). "An object-oriented approach for multi-objective flexible job-shop scheduling problem". Expert Systems with Applications, vol. 45, pp. 71-84 
Karimi, S.; Ardalan, Z.; Naderi, B. y Mohammadi, M. (2017). "Scheduling flexible job-shops with transportation times: Mathematical models and a hybrid imperialist competitive algorithm". Applied mathematical modellíng, vol. 41, pp. 667-682.

Liu, Y.; Dong, H.; Lohse, N. y Petrovic, S. (2015). "Reducing environmental impact of production during a Rolling Blackout policy - A multi-objective schedule optimisation approach". Journal of Cleaner Production, vol. 102, pp. 418-427.

Liu, Y.; Dong, H.; Lohse, N. y Petrovic, S. (2016). "A multiobjective genetical objective genetic algorithm for optimisation of energy consumption and shop floor production performance". Int. J. Production Economics, vol. 179, pp. 259-272.

Liu, Y.; Dong, H.; Lohse, N.; Petrovic, S. y Gindy, N. (2014). "An investigation into minimising total energy consumption and total weighted tardiness in job shops". Journal of Cleaner Production, vol. 65, pp. 87-96.

Ma, J.; Lei, Y.; Wang, Z.; Jiao, L. y Liu, R. (2014). "A memetic algorithm based on Immune multi-objective optimization for flexible job-shop scheduling problems". IEEE Congress on Evolutionary Computation (CEC), pp. 58-65.

Moon, J. y Park, J. (2014). "Smart production scheduling with time-dependent and machine-dependent electricity cost by considering distributed energy resources and energy storage". International Journal of Production Research, vol. 52, No. 13, pp. 39223939.

Niebel, W. y Freivalds, A. Ingeniería industrial: métodos, estándares y diseño del trabajo. 12 ed. México: Alfaomega, 2012. 258 p.

Niemi, E. y Pekkola, S. (2017). "Using enterprise architecture artefacts in an organisation". Enterprise information systems, vol. 11, No. 3, pp. 313-338.

Ojstersek, R.; Zhang, H.; Liu, S. y Buchmeister, B. (2018). "Improved Heuristic Kalman Algorithm for Solving Multi-Objective Flexible Job Shop Scheduling Problem". Procedia Manufacturing, vol. 17, pp. 895-902.

Pérez, R. y Hernández, A. (2018). "A hybrid estimation of distribution algorithm for flexible job-shop scheduling problems with process plan flexibility". Applied Intelligence, vol. 48, No. 10, pp. 3707-3734.

Rohaninejad, M.; Sahraeian, R. y Nouri, B. (2016). "Multiobjective optimization of integrated lot-sizing and scheduling problem in flexible job shops". Operations Research, vol. 50, No. 3, pp. 587-609.

Rudy, J. y Zelazny, D. (2014). “Solving multi-objective job shop problem using nature-based algorithms: new Pareto approximation features". An International Journal of Optimization and Control: Theories \& Applications, vol. 5, No. 1, pp. 1-11.

Ruiz, S.; Castrillón, O. y Sarache, W. (2012). "Una metodología multiobjetivo para optimizar un ambiente Job Shop". Información Tecnológica, vol. 23, No. 1, pp. 35-46.

Shen, X.; Zhang, M. y Fu, J. (2014). "Multiobjective Dynamic Job Shop Scheduling a Survey and Propects". International Journal of Innovative, vol. 10, No. 6, pp. 2113-2126.

Shivasankaran, N. (2015). "Hybrid sorting immune simulated annealing algorithm for flexible job shop scheduling". International Journal of Computational Intelligence Systems, vol. 8, No. 3, pp. 455-466.

Singh, M.; Singh, M.; Mahapatra, S. y Jagadev, N. (2016). "Particle swarm optimization algorithm embedded with maximum deviation theory for solving multiobjective flexible job shop scheduling problem". International Journal of Advanced Manufacturing Technology, vol. 85, pp. 2353-2366.

Yan, Y. y Yue, Y. (2015). "An improved genetic algorithm to the job shop scheduling problem". Chemical and Pharmaceutical Research, vol. 7, No. 4, pp. 322-325.

Wu, X. y Sun, Y. (2018). "A green scheduling algorithm for flexible job shop with energy-saving measures". Journal of Cleaner Production, vol. 172, pp. 32493264.

Zhu, C.; Qiu, W.; Zhu, M. y Chen, G. (2016). "Multi-objective flexible job shops robust scheduling problem under stochastic processing times". Mechanical Engineering Magazine Office, vol. 27, pp. 1667-1672.

PARA CITAR ESTE ARTÍCULO / TO REFERENCE THIS ARTICLE / PARA CITAR ESTE ARTIGO /

Coca Ortegón, G.A.; Castrillón Gómez, O.D.; Ruiz Herrera, S. (2019). Los grupos de interés en la programación de producción de un sistema de manufactura "Job Shop". Revista EIA, 16(32), Julio-Diciembre, pp. 65-84. [Online]. Disponible en: https://doi.org/10.24050/reia.v16i32.1236 